\title{
Management of Osteoporosis in The Community: How Well Can Family Physicians Do? A Retrospective Case Series in a Local Public Clinic in Hong Kong.
}

\section{Pui Kwan Chan ( $\triangle$ Chanjoanne825@gmail.com )}

Department of Family Medicine and General Outpatient Clinics (GOPCs), Kowloon Central Cluster (KCC), Hospital Authority (HA),Hong Kong

\section{Wing Shun, Vincent Li}

Department of Family Medicine and General Outpatient Clinics (GOPCs), Kowloon Central Cluster (KCC), Hospital Authority (HA),Hong Kong

\section{Yim Chu Li}

Department of Family Medicine and General Outpatient Clinics (GOPCs), Kowloon Central Cluster (KCC), Hospital Authority (HA),Hong Kong

\section{Xiao Rui, Catherine Chen}

Department of Family Medicine and General Outpatient Clinics (GOPCs), Kowloon Central Cluster (KCC), Hospital Authority (HA),Hong Kong

\section{Research Article}

Keywords: Family physician, osteoporosis, management outcome, T-score

Posted Date: December 31st, 2020

DOI: https://doi.org/10.21203/rs.3.rs-133928/v1

License: (c) (1) This work is licensed under a Creative Commons Attribution 4.0 International License. Read Full License 


\section{Abstract}

\section{Background}

With the ageing population, osteoporosis is increasingly becoming a global health concern. Previous research showed that management of osteoporosis in Hong Kong had been suboptimal. In view of the need to provide quality care to osteoporotic patients, a Multidisciplinary Osteoporosis Clinic (MOC) organized by primary care physicians was set up in a public primary care clinic. This study aimed to explore the clinical effectiveness of MOC.

\section{Methods}

We performed a retrospective case series study. All osteoporotic patients who had attended MOC during the period 1 January 2015 to 31 December 2018 were included. Changes in Dual X-ray absorptiometry (DEXA) T-score of recruited patients after two years of management at MOC were analysed. Subgroup analyses of the 2-year interval DEXA T-score changes among patient with or without history of fragility fracture, and among patients with or without pharmacological treatment, were performed. Serial interval DEXA T-score changes of recruited patients who had completed 5 years of bisphosphonate treatment were also analysed. Paired Student's t test was used to analyze the interval DEXA T-score changes.

\section{Results}

Totally 186 osteoporotic patients were recruited. After two years of management at MOC, the T-score at lumbar spine and femoral neck were improved, from $-2.71 \pm 0.76$ to $-2.35 \pm 0.83$ and $-2.40 \pm 0.75$ to $-2.10 \pm 0.76$ respectively, $P<0.001$. For subgroup analysis on patients with or without history of fragility fracture and patients with or without bisphosphonate treatment, improvement in T-scores of both lumbar and femoral neck were all significant at two years, $P<0.05$.

Among those who had completed 5 years of bisphosphonate treatment, progressive improvement in Tscores of both lumbar and femoral neck were observed, with $\mathrm{P}<0.001$.

\section{Conclusion}

MOC run by family physicians in public primary care setting could effectively treat osteoporotic patients with significant improvement in DEXA T-scores.

\section{Background}

With the ever-ageing population and longer life expectancy, osteoporosis is increasingly becoming a global health concern [1]. Currently it is estimated that more than 200 million people suffer from the disease worldwide [2]. Approximately $30 \%$ of all postmenopausal women have osteoporosis in the United State and in Europe. [3] According to a meta-analysis published in 2016, there has been an obvious increase in the prevalence of osteoporosis in China over the past 12 years (prevalence of $14.94 \%$ before 
2008 and $27.9 \%$ during the period spanning 2012-2015). The pooled prevalence of osteoporosis in people aged 50 years and older was $34.7 \%$, meaning around one third of people over 50 year-old are suffering from the disease [4]. In another local study, the prevalence of osteoporosis in Hong Kong Chinese female increases with age dramatically. In women aged 70 or above, over half have osteoporosis at the hip. [5]

Despite the enormous social and economic impact of osteoporosis and its related complication, namely fracture, osteoporosis remains underdiagnosed and undertreated worldwide. [6] A recent study conducted in Asia, including data from Hong Kong, highlighted the poor performance in osteoporosis diagnosis and management, even among high risk groups with prior fragility hip fractures. In the study, bone mineral density (BMD) measurement was only performed in $28.2 \%$ of patients prior to hip fractures; $51.5 \%$ were informed that they had osteoporosis after hip fractures, and $33.0 \%$ received medications for osteoporosis 6 months after discharge. [7]

According to Osteoporosis Society of Hong Kong, much of the responsibility for delivering effective treatment to osteoporotic patients falls on endocrinologists and orthopaedic surgeons in Hong Kong. With the long waiting time in specialty clinics, many patients miss the golden time for treatment, i.e. before the happening of fragility fracture and its associated morbidities and mortality. In view of this service gap, a dedicated Multidisciplinary Osteoporosis Clinic (MOC) was set up at a primary care clinic of Kowloon Centre Cluster (KCC) under the Hospital Authority of Hong Kong (HAHK) in 2010. The objective of setting up this clinic is to provide comprehensive management for osteoporotic patients in the primary care setting and to enhance community-based care. However, to date, studies are lacking in the evaluation of the clinical effectiveness of osteoporosis management by primary care physicians.

\section{Methods}

\section{Study design}

A retrospective case series study conducted in a public primary care clinic of KCC of HAHK.

\section{Setting of the Multidisciplinary Osteoporosis Clinic (MOC)}

Patients with confirmed osteoporosis or osteoporosis related conditions such as history of fragility fractures were referred to $\mathrm{MOC}$ for further management. The referring source was mainly from different general outpatient clinics (GOPCs) of KCC and other primary care providers in Hong Kong. This multidisciplinary clinic was specially designed to cater the needs of osteoporotic patients.

Patients referred to the clinic were invited to a Community Based Specialty Nursing Session which included a comprehensive osteoporosis management health talk and an individual counselling session. Fracture risk assessment tool (FRAX), a scoring system assessing one's 10-year osteoporotic fracture risk based on individual's clinical risk factors as well as BMD at the femoral neck [8], was used and FRAX score was calculated during the session. Advice was given accordingly. 
After the nursing educational session, a doctor consultation session was arranged in the following weeks. Doctors conducting the consultation acquired at least fellowship qualification of both Hong Kong College of Family Physicians (HKCFP) and Royal Australian College of General Practitioners RACGP (RACGP).

They were also equipped with updated knowledge regarding osteoporosis management. The consultation time was on average 15 minutes per patient, which was longer than the 7-minute consultation time in GOPC. This was to ensure sufficient time had been given to attending doctors to convey necessary pharmacological and non-pharmacological advice, and to provide quality medical assessment and management. [9]

Bisphosphonates are recommended as first line treatment in most guidelines including National Osteoporosis Foundation (NOF) guidelines and the American Association of Clinical Endocrinologists (AACE) guidelines $[10,11]$. Oral alendronate is the only pharmacological agent that can be reimbursed in HAHK for patients with history of osteoporotic fracture. Therefore, guideline directed medical therapy, namely alendronate was regularly prescribed in our clinic.

DEXA scan was performed upon joining MOC, i.e. baseline DEXA scan. It was repeated after 1-2 years of initiation of pharmacological treatment and every 2 years thereafter if BMD had been stabilized or improved, compatible with most guidelines' recommendation including NOF guidelines, AACE guidelines and the International Society for Clinical Densitometry (ISCD) guideline [10, 11, 12]. To ensure uniform data comparisons, patients were urged to have their DEXA scans repeated in the same diagnostic center, assuming the same DEXA machine was used. Doctor follow up appointment was arranged depending on clinical needs.

Allied health services such as dietitian counselling, physiotherapy and occupational therapy were also available upon doctor's referral.

\section{Participants}

All osteoporotic patients, coded by International Classification of Primary Care (ICPC) L95 (osteoporosis), who had attended MOC of KCC of the HAHK from 1 January 2015 to 31 December 2018.

\section{Exclusion criteria}

1. Osteopenia patients

2. Osteoporosis patients due to secondary causes such as osteoporosis due to endocrine diseases or corticosteroid use

3. Osteoporosis patients who had been followed by other specialists or private doctors

4. Osteoporosis patients who had been treated with osteoporosis medication before joining MOC, Osteoporosis medication means any type of bone antiresorptive and anabolic drugs, not including vitamin $\mathrm{D}$ and calcium supplement

5. Osteoporosis patients who had no baseline DEXA scan 
Definition of Osteoporosis

Dual X-ray absorptiometry (DEXA) is the gold standard and most precise technique for BMD measurement [13]. The difference between the patient's BMD and mean BMD of young female adult, divided by the standard deviation (SD) of the reference population, yields the T-score. A DEXA T-score of -1.0 or above was regarded as normal. While a DEXA T-score between -0.1 to -2.5 was regarded as osteopenia. Osteoporosis was defined as DEXA T-score of $\leq-2.5$, according to World Health Organization [14].

Determination of variables

The recruited patient's age, gender, ethnicity, smoking status, drinking status, body mass index (BMI), family history of fracture, past history of fracture, other past medical history or co-morbidities, menopausal status (for female), age of menopause, FRAX score were retrieved from nursing consultation notes of Clinical Management System (CMS) of HAHK. The BMI was calculated as body weight $(\mathrm{kg}) /$ body height ${ }^{2}\left(\mathrm{~m}^{2}\right)$. The patient was considered a smoker if he/she was an active smoker or had quit smoking within 6 months. Baseline blood test including complete blood count, liver function test, calcium level, phosphate level and thyroid function test of patients were collected from the CMS of HKHA.

Baseline and 2-year interval DEXA T-scores were collected. Baseline DEXA T-score was the T-score of DEXA scan performed upon joining MOC, within one year before or after first doctor consultation. The 2year interval DEXA T-score was the T-score of the DEXA scan performed at or nearest to 2 years after joining MOC. The serial interval T-scores of those who had completed 5 years of bisphosphonate were collected. These data were retrieved from doctor consultation notes from CMS of HKHA. If in any doubt, the original copy of DEXA report was referred to. The occurrence of new osteoporotic fractures documented in consultation notes was also recorded.

\section{Primary and Secondary outcome}

Primary outcome:

1. Changes in DEXA T-score of recruited osteoporotic patients after two years of management at MOC.

2. Subgroup analysis: 2-year interval DEXA T-score changes among patients with or without history of fragility fracture; and among patients with or without pharmacological treatment

3. Serial interval DEXA T-score changes of recruited patients who had completed 5 years of bisphosphonate

Secondary outcome: The occurrence of new osteoporotic fracture of recruited osteoporotic patients

\section{Sample size calculation}


From previous studies, the mean BMD difference between treatment and non-treatment group was 0.03 $\mathrm{g} / \mathrm{cm}^{2}$, with a SD of $0.13[15,16]$. At $95 \%$ confidence level and a power of 0.8 , with the use of paired sample $T$ test for sample size calculation, the sample size required is 147. To allow the room for data exclusion ( 20\%), totally 186 patients were included into the data analysis.

\section{Statistical analysis}

All data were entered and analyzed using computer software (SPSS version 23, Chicago, IL, US). Categorical variables were presented as frequencies and percentages. Continuous variables were presented as mean, plus standard deviation. Paired Student's $t$ test was used to analyze continuous variables. All statistical tests were two-sided, and a $P$ value of $<0.05$ is considered significant.

\section{Results}

A total of 507 patients with ICPC coding L95 (osteoporosis) attended MOC during the period 1/1/2015 to 31/12/2018. 321 patients were excluded while 186 patients were included in the final data analysis. Among the excluded, majority of them were excluded because they had been treated with osteoporosis medication by other doctors before joining MOC $(\mathrm{N}=160,50 \%)$ or they had no interval DEXA scan performed during the study period $(\mathrm{N}=102,32 \%)$. The flowchart of case recruitment is summarized in Figure 1.

Among the 186 patients included in the final data analysis, 182 were female (97.8\%) and 4 were male (2.2\%). 185 patients were of Chinese ethnicity. The mean age of the patients was $66.2+/-8.3$ years old. Majority of them were nonsmoker, nondrinker, with a mean BMI $21.5+/-3.1 \mathrm{~kg} / \mathrm{m}^{2}$. The demographics of the included patients are summarized in Table 1 a.

Table 1a Demographic characteristics of osteoporotic patients recruited into data analysis 


\begin{tabular}{|c|c|}
\hline Characteristics ł & $\mathrm{N}=186$ \\
\hline \multicolumn{2}{|l|}{ Sex } \\
\hline Female & $182(97.8 \%)$ \\
\hline Male & $4(2.2 \%)$ \\
\hline \multicolumn{2}{|l|}{ Ethnicity } \\
\hline Chinese & $185(99.5 \%)$ \\
\hline Indian & $1(0.05 \%)$ \\
\hline Age (years) & $66.2 \pm 8.3$ \\
\hline BMI $\left(\mathrm{kg} / \mathrm{m}^{2}\right)$ & $21.5 \pm 3.1$ \\
\hline Menopause age (Years) & $48.9 \pm 5.0$ \\
\hline \multicolumn{2}{|l|}{ Smoking status } \\
\hline Non smoker & $185(99.5 \%)$ \\
\hline Ex-Smoker & $1(0.05 \%)$ \\
\hline \multicolumn{2}{|l|}{ Drinking status } \\
\hline Non drinker & $185(99.5 \%)$ \\
\hline Drinker & $1(0.05 \%)$ \\
\hline \multicolumn{2}{|l|}{ Family history of fracture } \\
\hline Yes & $31(16.7 \%)$ \\
\hline No & $155(83.3 \%)$ \\
\hline \multicolumn{2}{|l|}{ Personal history of fracture } \\
\hline Yes & $39(21.0 \%)$ \\
\hline No & $147(79.0 \%)$ \\
\hline \multicolumn{2}{|l|}{ Pharmacological treatment } \\
\hline Yes & $153(82.3 \%)$ \\
\hline No & $33(17.7 \%)$ \\
\hline
\end{tabular}

Furthermore, 39 patients had personal history of fragility fracture (21.0\%). Among them, 15 suffered from spinal collapse (38.5\%), 9 had history of distal radius fractures $(23.1 \%), 4$ had proximal humerus fractures (10.2\%), 3 suffered from metatarsal fractures (7.7\%), and 2 suffered from hip fractures (5.1\%).

The mean baseline DEXA T-score of lumbar spine and femoral neck of the included 186 osteoporotic patients were $-2.71 \pm 0.76$ and $-2.40 \pm 0.75$ respectively. While the FRAX score was $10.3 \pm 8.0 \%$ for major osteoporotic fracture and $4.0 \pm 5.3 \%$ for hip fracture. The baseline T-score and FRAX score for different age groups of the included patients were summarized in Table $\mathbf{1 b}$.

Table 1b Baseline FRAX score and T-score of osteoporotic patients recruited into data analysis 


\begin{tabular}{|l|l|l|l|l|l|}
\hline Group & N=186 (\%) & $\begin{array}{l}\text { FRAX score \% } \\
\text { (major osteoporotic) }\end{array}$ & $\begin{array}{l}\text { FRAX Score \% } \\
\text { (hip) }\end{array}$ & $\begin{array}{l}\text { Lumbar spine T-score } \\
\text { (mean } \pm \text { SD) }\end{array}$ & $\begin{array}{l}\text { Femoral neck T-score } \\
\text { (mean } \pm \text { SD) }\end{array}$ \\
\hline Overall & $186(100 \%)$ & $10.3 \pm 8.0$ & $4 \pm 5.3$ & $-2.71 \pm 0.76$ & $-2.40 \pm 0.75$ \\
\hline Age $<50$ & $1(0.05 \%)$ & 1 & 0.1 & -2.50 & -1.40 \\
\hline Age 50-59 (years) & $46(24.7 \%)$ & $4.9 \pm 3.2$ & $1.4 \pm 1.7$ & $-2.61 \pm 0.79$ & $-2.20 \pm 0.60$ \\
\hline Age 60-69 (years) & $79(42.5 \%)$ & $9.6 \pm 6.6$ & $3.4 \pm 3.9$ & $-2.75 \pm 0.73$ & $-2.33 \pm 0.83$ \\
\hline Age 70-79 (years) & $47(25.3 \%)$ & $12.7 \pm 4.5$ & $4.9 \pm 2.7$ & $-2.71 \pm 0.72$ & $-2.49 \pm 0.61$ \\
\hline Age $>80$ (years) & $13(7.0 \%)$ & $24.8 \pm 14.4$ & $14.3 \pm 11.6$ & $-2.82 \pm 1.00$ & $-3.05 \pm 0.83$ \\
\hline
\end{tabular}

*Data are show as No., No,(\%), and mean \pm standard deviation

Baseline blood test showed 12 out of 186 patients had anaemia. 6 patients were thalassemia carrier, 1 suffered from per rectal bleeding, and the remaining 5 had no identified cause for the anaemia. The baseline liver, calcium, phosphate and thyroid hormone levels were normal.

After 2 years of management in MOC, T-scores of recruited patients improved from $-2.71 \pm 0.76$ to $-2.35 \pm 0.83$ at lumbar spine, and from $-2.40 \pm 0.75$ to $-2.10 \pm 0.76$ at femoral neck, which were both statistically significant, $P<0.001$. The results are shown in Figure 2 .

Among the 186 patients, 146 patients (78.5\%) had T-score improvement at lumbar spine and 124 patients (66.7\%) had T-score improvement at femoral neck. 142 patients were osteoporotic in lumbar spine before joining MOC and 53 of them (37.3\%) improved to osteopenia range at 2-year follow up; while 106 patients were osteoporotic in femoral neck and 49 of them (46.2\%) became osteopenia after 2 year of management in MOC.

Subgroup analysis of patients with history of fragility fracture showed that the baseline T-scores were lower at both lumbar and femoral neck region compared to those without history of fragility fracture, which were $-2.96 \pm 0.77$ and $-2.57 \pm 0.72$ respectively. Improvement in T-scores of both lumbar $(P<0.001)$ and femoral neck $(P=0.0017)$ were significant at 2-year follow up. For those without history of fragility fracture, improvement in T-score of both lumbar and femoral neck were also observed with $\mathrm{P}<0.001$. Summary of this subgroup analysis results are shown in Figure 3.

Among the 186 patients, 153 of them (82.7\%) received osteoporosis drug treatment, namely bisphosphonate, in MOC. While 33 of them (17.3\%) had not received drug treatment and was on life style modifications. Subgroup analysis of those who received pharmacological treatment showed significant improvement in T-scores of both lumbar and femoral neck, $P<0.001$. While those who was on lifestyle modifications without drug treatment also enjoyed T-scores improvement at both lumbar spine $(P<0.001)$ and femoral neck $(P=0.025)$. The results of this subgroup analysis are summarized in Figure 4. 
During the follow up period, 46 patients received and completed 5 years of bisphosphonate treatment. Progressive improvement in T-scores of both lumbar and femoral neck were observed, with the mean Tscore of lumbar spine improving from $-2.79 \pm 0.73$ to $-2.22 \pm 0.74(P<0.001)$ and that of femoral neck improving from $-2.48 \pm 0.60$ to $-1.93 \pm 0.74(P<0.001)$. Results of the interval T-scores of lumbar spine and femoral neck during 5 years of drug treatment are shown in Figure 5.

Regarding the secondary outcome, 1 out of the 186 osteoporotic patients suffered from new osteoporotic fractures among the 2-year follow-up period. The incidence rate was 2.7 cases per 1000 person-years. This patient suffered from left hip fragility fracture after two years of oral alendronate.

\section{Discussion}

To our knowledge, no similar team based management model in primary care for osteoporotic patients has been described. In this retrospective case series analysis, we demonstrated that a dedicated team led by primary care physicians could significantly improve the bone mineral density of osteoporotic patients. Our patient demographics are in line with that of other studies on osteoporotic patients conducted in china and Asia. $[17,18,19]$, with a mean age of 66.1 and a majority of them being post-menopausal women with menopause at a mean age of 48.9. The results of our study were very promising that DEXA T-scores of the recruited patients improved significantly in both lumbar and femoral neck, $P<0.001$. Over $80 \%$ of osteoporotic patients joining the $\mathrm{MOC}$ were started on osteoporosis drug treatment which was significantly higher than the treatment rate of previous studies [7]. For patients with pharmacological treatment, predominately treated with alendronate, their 2-year interval T-score improved 0.38SD (from -2.76 to -2.38 ) at lumbar spine and 0.33 SD (from -2.45 to -2.12 ) at femoral neck, which were comparable with other published clinical studies [20,21,22]. Furthermore, subgroup analysis of patients who were not on drug treatment also showed significant improvement in T-scores of both lumbar and femoral neck. This suggests that the T-score changes and BMD improvements could not be solely explained by the pharmacological effects of bisphosphonates but also by the comprehensive services provided by the MOC.

The reasons why this $\mathrm{MOC}$ is highly effective is multi-factorial. First of all, the MOC is a well-designed clinic tailored to manage osteoporotic patients. There is a protocol for staff to refer to and follow once the patients attend the clinic. Secondly, our nurse would meet every patient during the individual counselling session when they would collect information on patients' demographics and calculate the FRAX scores and give individualised advice accordingly. Thirdly, Doctors who have attended the MOC are relatively experienced family physicians who have achieved intermediate family Medicine training qualifications. In addition, a longer consultation time (around 15 minutes per case) would also allow the doctor to discuss the management plan with the patients more thoroughly compared with GOPC settings (around 6-7 minutes per case). Indeed, previous research on the doctors' barriers on osteoporosis treatment have revealed that a lack of knowledge on osteoporosis drug treatment and specifically its adverse effects may deter many doctors from treating the disease $[23,24]$. By centralizing this group of 
osteoporotic patients to MOC with dedicated doctors and nurses, many of these obstacles of care could be overcome, resulting in improvements in outcomes.

This study has several strengths. This is the first study ever in primary care of Hong Kong to evaluate the effectiveness of a community based osteoporosis clinic, in terms of DEXA T-score changes in osteoporotic patients. Previous studies published mainly focused on the detection and treatment rate of osteoporosis $[6,7,25]$, or tried to explore factors preventing the delivery of effective osteoporosis treatment to osteoporotic patients $[7,26]$. Our study is comprehensive and impactful in terms of the outcome measurements. It not only assessed the treatment rate of osteoporotic patients but also the changes in their BMD and the occurrence of fall during the follow up period, which are importance parameters directly influencing the mortality and morbidity brought about by osteoporosis. Another strength of the study is that the clinical data were precisely documented and complete. In MOC, nurses would document patients' epidemiological data, using a preset template, in the nursing notes of CMS of HAHK. Doctors would also document patients' clinical condition, DEXA scan and blood investigation reports in consultation notes of CMS with a similar format. These measures minimized the possibility of missing data or recall bias.

There are some limitations in this study. Firstly, a large proportion of patients were excluded from the final data analysis, which might result in selection bias. Majority of the patients excluded had been treated with bone resorptive agents before joining MOC $(\mathrm{N}=160)$ or they had no interval DEXA scan within the follow up period $(N=102)$. Bone resorptive agents could have lasting effects on residual BMD and fracture prevention despite stopping the drug $[27,28]$. Patient's DEXA T-score change after joining MOC could be related to previous treatment instead of the true effect of MOC, therefore were excluded. we believe this treatment naive group of patient could provide a fair reflection of the effectiveness of MOC. Secondly, this study was carried out in a single public primary care clinic in HAHK and therefore, the results of this study may not be generalized to all primary care clinics in Hong Kong or in the private setting. In addition, most patients followed up in MOC had to pay for their own drugs and DEXA scans as they were not readily available from HAHK for free, except for osteoporotic patients with past history of fragility fracture. Therefore, those who continued to follow-up in MOC could well represent a group of patients who were more health conscious and financially capable. Thirdly, since this is a retrospective study without control group, we should be cautious in attributing all the positive outcome to the effect of MOC. However, available literature has reported that age-related T-score of Chinese women who are treatment naive deteriorates with age [29]. Hence, despite the lack of a control group, in view of the significant improvements in T-score of patients who joined the MOC, especially in the non-pharmacological group, it is justifiable to credit improved BMD of osteoporotic patients to the effective management at MOC.

\section{Conclusion}

With its growing incidence and increasingly significant complications, osteoporosis represents a major burden to healthcare. This study provided invaluable evidence that a multidisciplinary osteoporosis clinic run by dedicated family physicians in a public primary care setting can effectively treat osteoporosis 
patients with significant improvement in T-scores. We believe that, based on the findings of this study, an integrated, comprehensive and multi-disciplinary service model could be developed in primary care to meet the service demand in managing osteoporosis patients in the community.

\section{Abbreviations}

AACE: American Association of Clinical Endocrinologists

BMD: Bone Mineral Density

BMI: Body Mass Index

CMS: Clinical Management System

DEXA: Dual X-ray Absorptiometry

FRAX: Fracture Risk Assessment tool

GOPCs: General Outpatient Clinics

HAHK: Hospital Authority of Hong Kong

HKCFP: Hong Kong College of Family Physician

ICPC: International Classification of Primary Care

ISCD :International Society for Clinical Densitometry

KCC: Kowloon Central Cluster

MOC: Multidisciplinary Osteoporosis Clinic

NOF: National Osteoporosis Foundation

RACGP: Royal Australian College of General Practitioners

SD: Standard Deviation

\section{Declarations}

\section{Ethics approval and consent to participate}

This study have been performed in accordance with the Declaration of Helsinki.

This study was approved by the Hong Kong Kowloon Center/ Kowloon East Cluster Research Ethics Committee, with reference number: KC/KE-18-0183/ER-1. 
Since this is a retrospective case series, only the medical records of the recruited cases within the CMS of HAHK were reviewed, that their clinical management was not affected, neither verbal nor written consent to participate in the study or to publish the study is required, according to the regulations of Research Ethics Committee of KCC of HAHK.

\section{Availability of data materials}

The datasets used in the current study were compiled by the corresponding author. They would be available from the corresponding author on reasonable request after being approved by head of department.

\section{Author information}

\section{Affiliations}

Department of Family Medicine and General Outpatient Clinics (GOPCs), Kowloon Central Cluster (KCC), Hospital Authority (HA), Hong Kong

P. K. Chan, W. S. Vincent Li, Y.C.Li, X. R. Catherine Chen

\section{Contributions}

All authors (CPK, LYC, LWCV, CXRC) had full access to the data, contributed to the study, approved the final version of publication, and taken responsibility for its accuracy and integrity. Authors (CPK, CXRC) contributed to the concept and design of the study. Authors (CPK, CXRC) contributed to the acquisition of data, analysis or interpretation of the data, drafting the manuscript and critical revision for important intellectual content.

Corresponding author

Correspondence to P. K. Chan.

Email: chanjoanne825@gmail.com

\section{Acknowledgements}

We would like to express our sincere gratitude to all medical staff and nursing staff for their professional service at MOC of KCC of HAHK.

\section{Funding}

This research project was not funded by any agency in the public, commercial, or not-for-profit sectors.

\section{Consent for publication}

No applicable. 


\section{Competing interests}

All authors have declared no competing interests.

\section{References}

1. Reginster JY. Burlet N. Osteoporosis: A still increasing prevalence. Bone 2006;38: S4-S9.

2. Cooper C, Campion G, Melton LJ 3rd. Hip fractures in the elderly: a world-wide projection. Osteoporos Int. 1992 Nov;2(6):285-9.

3. Melton LJ, III, Chrischilles EA, Cooper C,et al. Perspective: How many women have osteoporosis? J Bone Miner Res. 1992;7:1005-10.

4. Peng Chen, Zhanzhan Li, and Yihe Hu. Prevalence of osteoporosis in China: a meta-analysis and systematic review. BMC Public Health 16; 1039.

5. Ho SC, Lau EM, Woo J, et al. The prevalence of osteoporosis in the Hong Kong Chinese female population. Maturitas. 1999 Aug 16;32(3):171-8.

6. Elliot-Gibson V, Bogoch ER, Jamal SA, et al. Practice patterns in the diagnosis and treatment of osteoporosis after a fragility fracture: a systematic review. Osteoporos Int. 2004;15:767-778.

7. A.W. Kung, T. Fan, L. Xu, et al. Factors influencing diagnosis and treatment of osteoporosis after a fragility fracture among postmenopausal women in Asian countries: a retrospective study. BMC Womens Health 2013 Feb 14;13:7.

8. FRAX, WHO fracture risk assessment tool. https://www.sheffield.ac.uk/FRAX/. Accessed 31 March 2019.

9. Freeman GK, Horder JP, Howie JG, et al. Evolving general practice consultation in Britain: issues of length and context. BMJ. 2002 Apr 13; 324(7342):880-2.

10. Cosman F, de Beur SJ, LeBoff MS, et al. Clinician's Guide to Prevention and Treatment of Osteoporosis. Osteoporos Int 2014; 25:2359.

11. Pauline M. Camacho, Steven M. Petak, Neil Binkley, et al. American association of clinical endocrinologists and American college of Endocrinology clinical practice guidelines for the diagnosis and treatment of postmenopausal osteoporosis 2016. Endocrine practice Vol 22 (suppl 4) September 2016.

12. 2013 ISCD Official Positions - Adult. https://iscd.org/learn/official-positions/adult-positions/. Accessed 1 March 2019.

13. Blake GM, Fogelman I. The role of DXA bone density scans in the diagnosis and treatment of osteoporosis. Postgrad Med J. 2007;83:509-17.

14. World Health Organization (2007) Assessment of osteoporosis at the primary health care level. Summary Report of a WHO Scientific Group. WHO, Geneva.

15. Liberman UA, Weiss SR,et al. Effect of oral alendronate on bone mineral density and the incidence of fractures in postmenopausal osteoporosis. The Alendronate Phase III Osteoporosis Treatment Study 
Group. N Engl J Med. 1995 Nov 30;333(22):1437-43.

16. Chan W. P., Liu J. F. \& Chi W. L. Evaluation of bone mineral density of the lumbar spine and proximal femur in population-based routine health examinations of healthy Asians. Acta Radiol. 45, 59-64 (2004).

17. Suzanne C. Ho, Edith M.C. Lau, Jean Woo, et al. The prevalence of osteoporosis in the Hong Kong Chinese female population. Maturitas. 1999 Aug 16;32(3):171-8.17 .

18. Tian L1, Yang R, Wei L, et al. Prevalence of osteoporosis and related lifestyle and metabolic factors of postmenopausal women and elderly men: A cross-sectional study in Gansu province, Northwestern of China. Medicine (Baltimore). 2017 Oct;96(43):e8294.

19. Hyun Koo Yoon,Young-Kyun Lee, and Yong-Chan Ha. Characteristics of Patients Diagnosed with Osteoporosis in South Korea: Results from the National Claim Registry. Bone Metab. 2017 Feb; 24(1): 59-63.

20. Rozkydal Z, Janicek P. The effect of alendronate in the treatment of postmenopausal osteoporosis. Bratisl Lek Listy. 2003;104(10):309-13.

21. Rizzoli R, Greenspan SL, Bone G $3^{\text {rd }}$, et al. Two-year results of once-weekly administration of alendronate $70 \mathrm{mg}$ for the treatment of postmenopausal osteoporosis. J Bone Miner Res. 2002 Nov;17(11):1988-96.

22. Ho AY, Kung AW. Efficacy and tolerability of alendronate once weekly in Asian postmenopausal osteoporotic women. Ann Pharmacother. 2005 Sep;39(9):1428-33.

23. Simonelli C, Killeen K, Mehle S, et al. Barriers to osteoporosis identification and treatment among primary care physicians and orthopaedic surgeons. Mayo Clini Proc. 2002 Apr; 77(4): 334-8.

24. Mendis AS, Ganda K, Seibel MJ. Barriers to secondary fracture prevention in primary care. Osteoporosis Int 2017 Oct;28(10):2913-2919.

25. Stephen H. Gehlbach, Maureen Fournier, Carol Bigelow, etal. Recognition of Osteoporosis by Primary Care Physicians. Am J Public Health 2002 February; 92(2)271-273.

26. Ip TP, Lam CLK, Kung AWC. Awareness of osteoporosis among physicians in China. Osteoporos Int 2004;15:329e34.

27. Black DM, Schwartz AV, Ensrud KE, et al. Effects of continuing or stopping alendronate after 5 years of treatment: the Fracture Intervention Trial Long-term Extension (FLEX): a randomised trial. JAMA 2006;296(24):2927.

28. Black DM, Delmas PD, Eastell R, et al. Once yearly zoledronic acid for treatment of postmenopausal osteoporosis. NEJM2007;356(18);1809.

29. Er-Yuan Liao, Xian-Ping Wu, Diao-Ge Deng, et al. Age-related bone mineral density, accumulated bone loss rate and prevalence of osteoporosis at multiple skeletal sites in Chinese women. Osteoporosis Int (2002) 13:669-676.

\section{Figures}


Total 507 patients, coded (ICPC) L95 (osteoporosis), were recruited from MOC from period 1/1/2015-31/12/2018

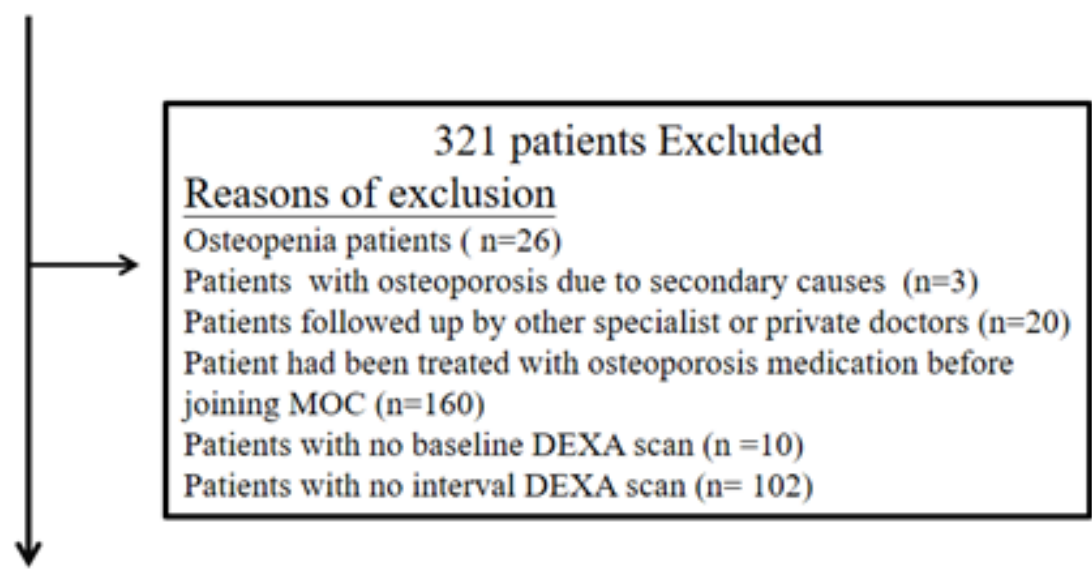

186 patients included in final data analysis

\section{Figure 1}

Flowchart of case recruitment for the study

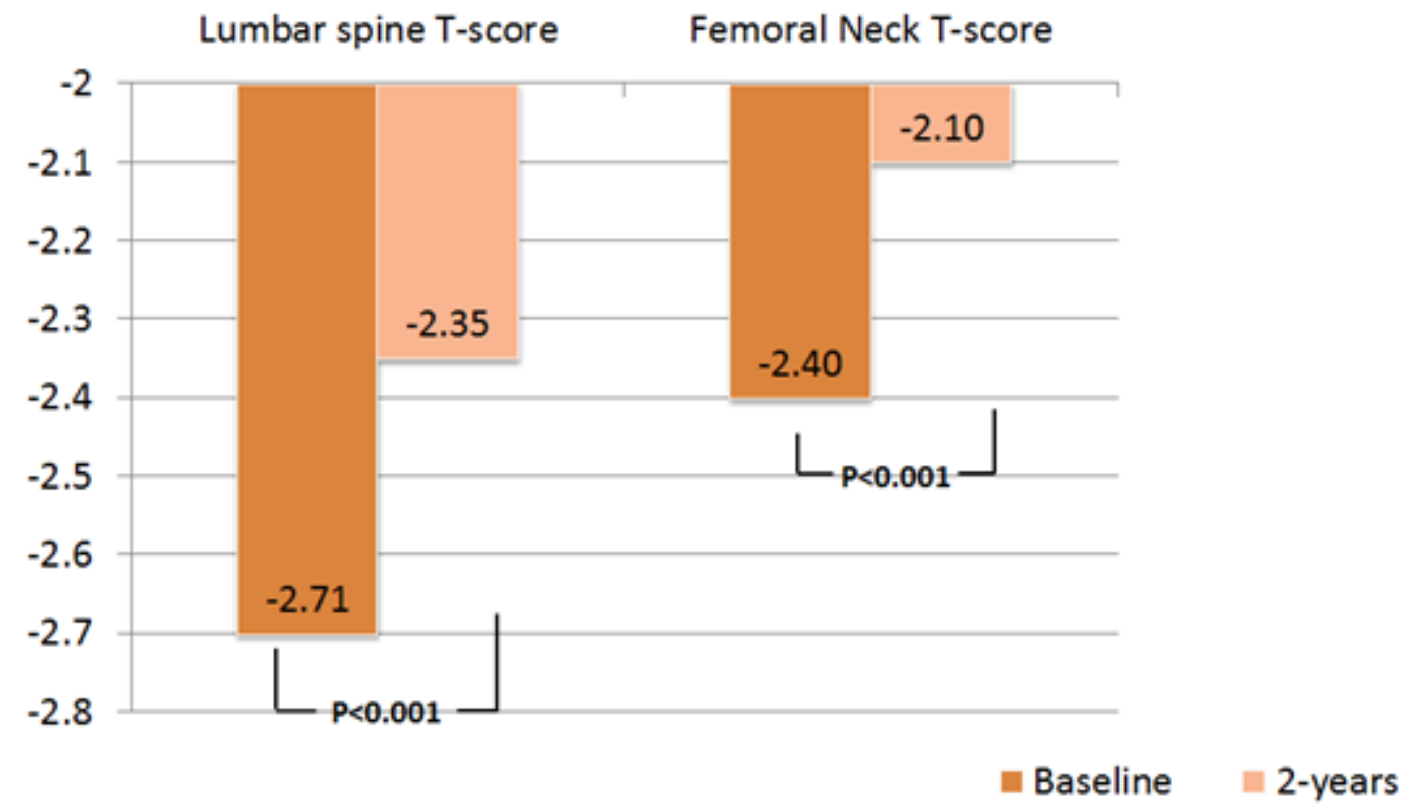

Figure 2

DEXA T-scores of recruited patients at baseline and 2 years after follow up in MOC 

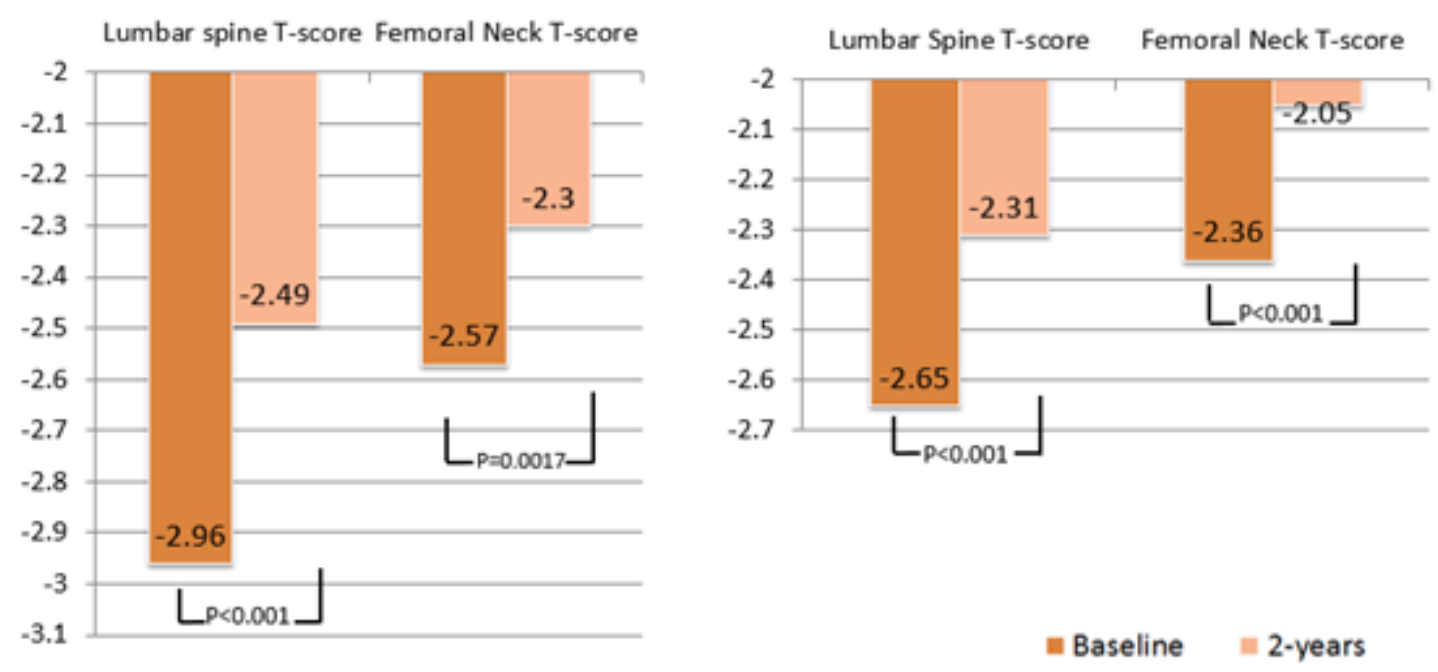

Figure 3

DEXA T-scores of recruited patients with (left side) and without (right side) history of fragility fracture at baseline and 2-year follow up at MOC
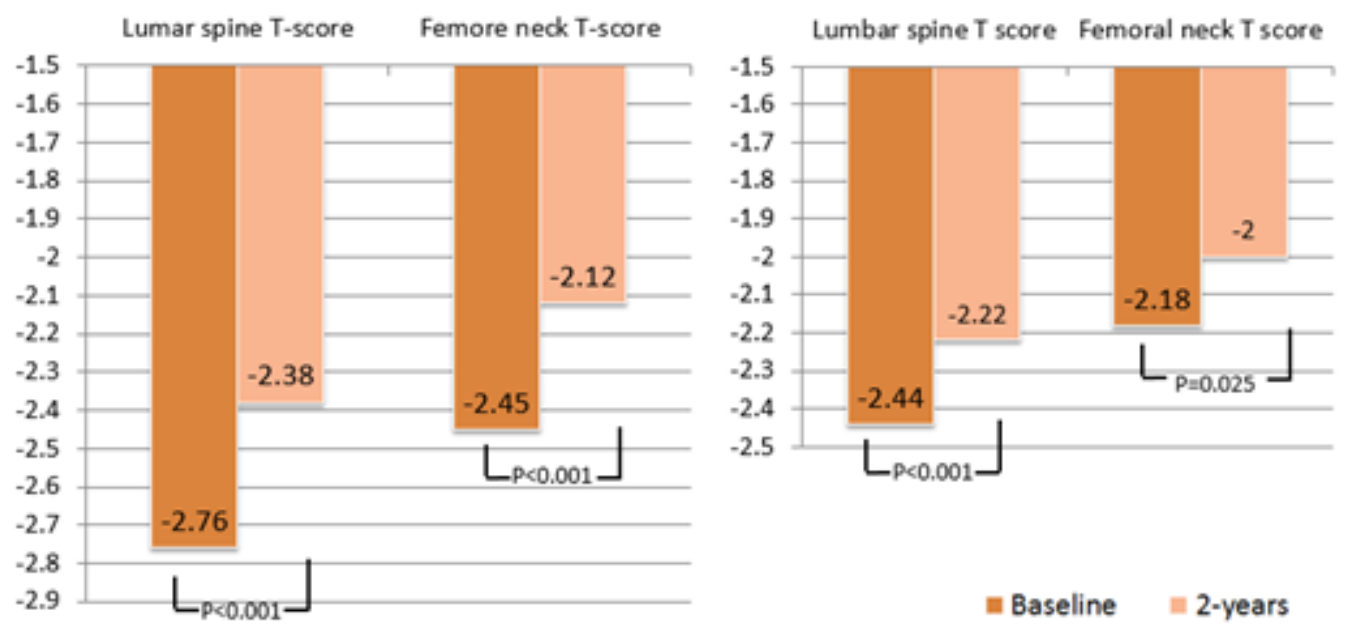

\section{Figure 4}

DEXA T-scores of recruited patients with (left side) and without (right side) drug treatment at baseline and 2 years after follow up in MOC 


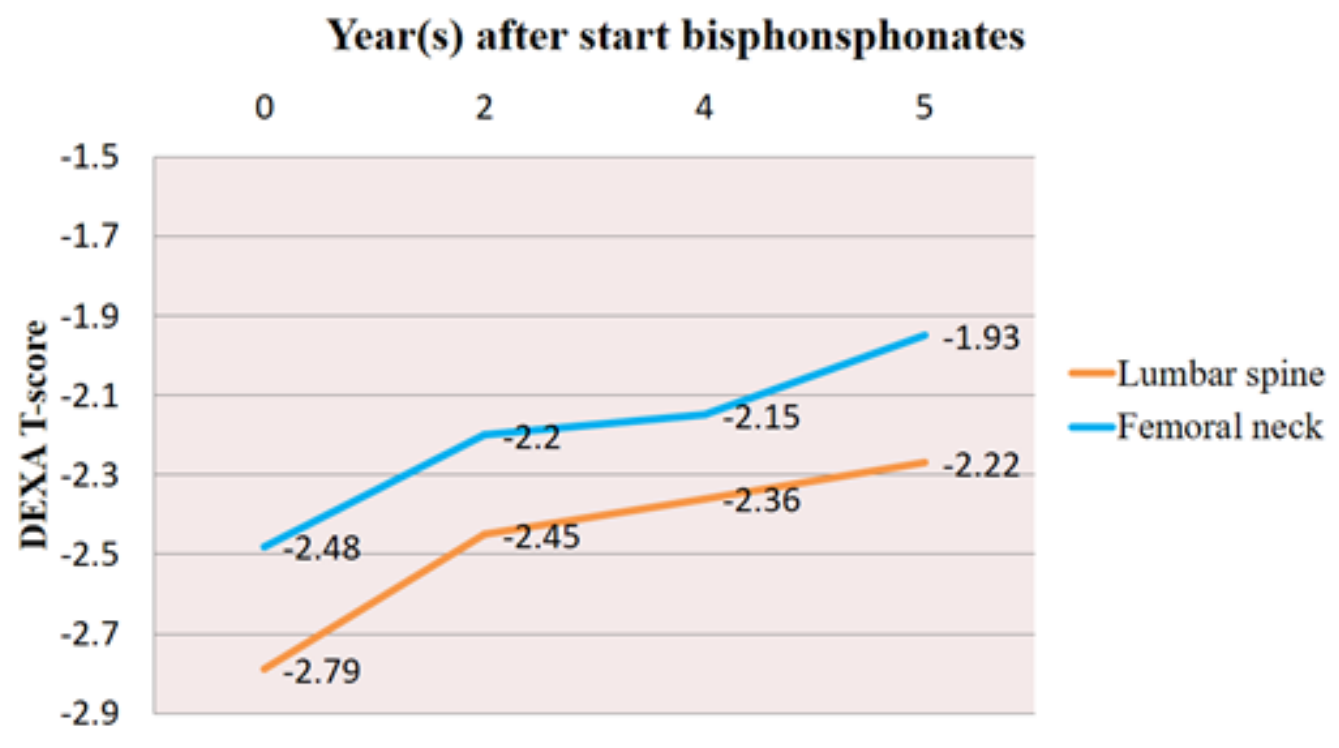

Figure 5

Interval T-scores of lumbar spine and femoral neck of patients who completed 5 years of bisphosphonate treatment 